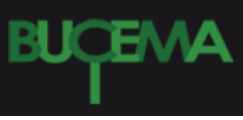

Bulletin du centre d'études médiévales d'Auxerre | BUCEMA

Hors-série $n^{\circ} 2 \mid 2008$

Le Moyen Âge vu d'ailleurs

\title{
Causa, creencia y testimonios. La prueba judicial en Castilla durante el siglo XIII
}

\section{Marta Madero}

\section{(2) OpenEdition}

1 Journals

\section{Edición electrónica}

URL: https://journals.openedition.org/cem/9672

DOI: $10.4000 /$ cem. 9672

ISSN: 1954-3093

\section{Editor}

Centre d'études médiévales Saint-Germain d'Auxerre

\section{Referencia electrónica}

Marta Madero, "Causa, creencia y testimonios. La prueba judicial en Castilla durante el siglo XIII », Bulletin du centre d'études médiévales d'Auxerre I BUCEMA [En ligne], Hors-série n 2 | 2008, mis en ligne le 19 janvier 2009, consulté le 03 mars 2023. URL : http://journals.openedition.org/cem/9672 ; DOI : https://doi.org/10.4000/cem.9672

\section{Este documento fue generado automáticamente el 3 marzo 2023}

\section{c) (i) (2)}

Creative Commons - Attribution - Pas d'Utilisation Commerciale - Partage dans les Mêmes Conditions 4.0 International - CC BY-NC-SA 4.0

https://creativecommons.org/licenses/by-nc-sa/4.0/ 


\title{
Causa, creencia y testimonios. La prueba judicial en Castilla durante el siglo XIII ${ }^{1}$
}

\author{
Marta Madero
}

1 En los últimos años, la interrogación sobre las técnicas y los procedimientos que permiten a una comunidad dar por válidos los enunciados que produce y considerarlos, por lo tanto, "verdaderos", ha conducido a una profunda renovación de varias disciplinas clásicas ${ }^{2}$. Baste recordar las discusiones abiertas sobre la relación - negada o afirmada según los casos - entre figuras del discurso y pruebas factuales en los estudios consagrados a la retórica clásica ${ }^{3}$, o los trabajos de la nueva historia de las ciencias sobre las formas, a menudo contradictorias, de los criterios de conocimiento utilizados para descifrar las leyes que gobiernan el mundo natural ${ }^{4}$. La historia misma, en tanto disciplina de saber, ha sido sometida a los mismos cuestionamientos sobre las relaciones entre su escritura, que necesariamente maneja las figuras y las formas de los relatos de ficción y la ambición que es la suya : decir adecuadamente, en el presente, una realidad que fue y ya no es ${ }^{5}$. Estas interrogaciones formularon de manera nueva problemas tradicionales de la epistemología de los saberes poniéndolos en relación con la diversidad y la especificidad de los regímenes de prácticas (discursivas o no) que constituyen las experiencias, a la vez compartidas y singulares, de una sociedad dada. Es en este marco de reflexión que quisiera inscribir las reflexiones que propongo sobre las formas de la prueba judicial y la reconstrucción de los hechos en la Castilla medieval ${ }^{6}$, esencialmente durante el siglo XIII -aunque la selección de fuentes hace desbordar la reflexión hacia la Castilla foral del fin del XII, por una parte, y hasta mediados del siglo XVI, por otro, dado que incluye los comentarios a las Siete Partidas de Gregorio López de 1555.

2 Uno de los mayores fenómenos intelectuales de la historia del Occidente medieval es el de la recepción del derecho romano a partir de fines del siglo XI, momento en que Irnerio comienza en Bolonia la reconstrucción del corpus jurídico justinianeo ${ }^{7}$. La reflexión doctrinal que se elabora en torno a estos textos fundadores, que muy 
rápidamente se desplaza fuera de Italia ${ }^{8}$, representa un inmenso trabajo intelectual que permite pautar un discurso normativo fuertemente común al espacio europeo ${ }^{9}$. Asimismo, impone una serie de modificaciones en diversos registros de racionalidad, particularmente en la lógica que rige la reconstrucción de los hechos en el espacio judicial. Pero las mutaciones exceden a las prácticas de establecimiento de la verdad, afectan a los modos de inculpación y denuncia - pasaje de las formas acusatorias a las formas inquisitivas del proceso ${ }^{10}$-, a las jerarquías delictivas - énfasis en las relaciones entre herejía y lesa majestad ${ }^{11}$-, a la consolidación de la memoria institucional abandono de las prácticas estrictamente orales en favor de la escritura ${ }^{12}-$, al rol que adquiere el castigo en la construcción del Estado ${ }^{13}$.

3 Las reflexiones que propongo intentan ser una contribución a sólo uno de estos temas. El problema teórico y práctico que está en el centro de la investigación es el de la construcción de la verdad judicial. Se trata de saber de qué modo, a partir de los testimonios o de otras formas de prueba, se reconstruyen « hechos » de forma tal que se pueda pronunciar una sentencia. Esto exige, a la vez, una reflexión sobre la creencia, los criterios sociales de la credibilidad, las formas de constitución del espacio judicial, la figura del juez y los modos de la argumentación. Se trata de explorar una tensión fundamental. Por una parte, el lugar y los procedimientos de la justicia movilizan saberes, representaciones, que comparten con otros regímenes de prácticas, desde la jerarquización de los sentidos a las figuras del discurso o los criterios de conocimiento. Por otra parte, la obra de justicia se despliega según lógicas propias, a través de categorías que se sitúan a distancia de las evidencias del mundo sensible produciendo « verdades » que se apoyan en operaciones intelectuales complejas y específicas.

El lenguaje jurídico, como el latín neoclásico de los humanistas, «no fue jamás concebido como la formulación anhelante de las percepciones frescas del mundo ${ }^{14}$ ». Como todo lenguaje, el de los juristas «es una conjura contra la experiencia, una empresa colectiva de simplificación y disposición que tiende a transformar la experiencia en elementos manejables ${ }^{15} »$. Se trata por lo tanto de entender ciertas reglas de funcionamiento que ordenan la experiencia en el espacio judicial. Como sucede con toda norma, saber que no siempre es cumplida, que es susceptible de interpretaciones varias, no implica que no diga nada sobre la sociedad que la produce error que a nuestro entender ocurre cuando se realiza un análisis de la práctica judicial que favorece estrictamente una lectura en términos de resolución de conflictos ${ }^{16}$. La alta tecnicidad del lenguaje jurídico obliga a reformular la experiencia, califica los hechos e impone condiciones de veridicción que los textos que presentamos procuran entender. La experiencia no es, dice Joan W. Scott, «el origen de nuestra explicación ${ }^{17}$, no traduce la idea de una transparencia plena, a la vez a lo real y a los sujetos. Lo que pretendo mostrar es por el contrario el encuentro entre modelos de comprensión y enunciación de lo real que organizan la posibilidad de la verdad. Estudios recientes sobre el proceso han puesto de manifiesto otros aspectos de la institucionalización de los hechos y han reflexionado sobre los efectos de las normas sobre la verdad y la experiencia. De la construcción de la fama a la aplicación de la tortura, la institución termina por "arrancar » una verdad que ella misma, en gran medida, formula. La tortura es sin duda la más brutal de estas estrategias, pero aún en el proceso civil es posible detectar las marcas que organizan las verdades de los hechos.

5 La investigación se ordena en tres preguntas sucesivas. La primera concierne un efecto del lenguaje : la construcción discursiva de lo real, siguiendo el modelo de los ordines 
romano-canónicos. La segunda, centrada en el problema de la creencia, procura indicar los modos plurales del "creer verdadero ", cruciales en un espacio judicial en donde todo depende de la creencia del juez, fundada en la credibilidad de los testigos que, a su vez, pueden ver, pero también creer que lo que dicen es fundado o cierto. La tercera pregunta nos conduce a un trabajo sobre las categorías intelectuales y las jerarquías sociales que son la matriz de los criterios de certificación y autentificación de las diferentes formas de prueba.

Causa y prueba, o cómo se perciben los hechos

El corpus sobre el que he trabajado está constituído por un conjunto de fueros ${ }^{18}$, una recopilación privada de la segunda mitad del siglo XIII - el Libro de los Fueros de Castiella ${ }^{19}$ - y de la obra jurídica del rey Alfonso X, escrita entre los años cincuenta y ochenta del siglo XIII ${ }^{20}$.

7 No me detendré aquí en una comparación sistemática entre fueros y obra del rey Sabio. Lo que me interesa marcar es la diferencia más importante entre los fueros y el grupo Espéculo-Siete Partidas - el Fuero Real se mantiene más próximo a la tradición foral -; esta reside en la creación de un método, de un marco abstracto en cuyo interior muchas operaciones continúan existiendo, pero formalizadas de otro modo, enmarcadas por los diferentes momentos de un debate y por una serie de distinciones que fragmentan y recomponen los enunciados que intentan dar cuenta de la experiencia inmediata. El Espéculo y las Partidas coloca la práctica judicial en otro marco lógico, incluso si los recursos probatorios no conocen cambios radicales. Pero este cambio es fundamental, puesto que la calificación y la posición de la causa como condiciones del conocimiento, producen una relación particular a lo real.

8 En un libro importante, Alessandro Giulani ${ }^{21}$ estableció la manera en que la prueba se torna un argumentum, a partir del momento en que el pensamiento jurídico adopta la noción de status, elaborada en la retórica de Hermágoras de Temno (I a.C.), que se conoce por testimonios indirectos: el De inventione de Cicerón, la Rhetorica ad Herennium, las Institutiones de Quintiliano y la Rhetorica que fue atribuida a san Agustín.

El status establece un centro de argumentación, el lugar de una controversia (feciste, non fecit) que determinará la pertinencia de los medios de prueba excluyendo todos los que no son pertinentes para la causa, tal como ha sido formulada luego de la serie de distinciones que la constituyen ${ }^{22}$. Según el testimonio de Quintiliano, para Hermágoras, el status « est ad quem probationes partium referantur ${ }^{23}$ ». El término argumentum será, sin embargo, apartado de algunas definiciones medievales de la prueba que preferirán una enumeración de los medios de prueba de la que se excluya una palabra que evoca demasiado la persuasión retórica. En esta perspectiva, lo que debe contar son las prueba que contengan rastros del hecho, la impronta de lo real. Pero la construcción discursiva de los « hechos » es previa a la prueba, se formula ya en la litis contestatio y en la teoría de las positiones. Veamos como las explica un texto procesal en lengua vernácula del último tercio del siglo XIII, es decir, contemporáneo de las fases finales de la obra alfonsina.

La Margarita de los pleitos ${ }^{24}$, texto procesal escrito después de 1263, ofrece una serie de consejos sobre las palabras que se deben usar para "façer las posiciones". Las positiones, que tienen lugar luego de la litis contestatio, es decir, luego del momento de aceptación del proceso por parte de los litigantes, son una serie de frases, de proposiciones que una parte afirma y a las cuales el adversario debe responder de forma negativa o afirmativa. Esta fase es un momento fundamental de definición de los 
« hechos » de la que no participa el juez, en razón del principio según el cuál judex non potest de facto supplere, es decir, queda excluida toda intervención del juez en las cuestiones de facto ${ }^{25}$. Cuantas más proposiciones sean confirmadas por el adversario, más credibilidad adquiere la versión del actor. Una demanda demasiado genérica que autoriza una negación absoluta es desaconsejada en la medida en que anula todos los elementos del enunciado.

11 Así, explica el texto, si mi intención es probar que tú estás excomulgado porque has herido a un clérigo y que lo probaré por «posiçiones", pondré así: "que feriste a Pedro clérigo tal dia », si tú lo niegas, yo podré entonces decir "pongo que feriste a Pedro, e non digo clérigo ", si tú aceptas que has herido a Pedro, entonces bien digo después "que aquel Pedro que tú feriste que era clerigo », si tú lo "otorgas », habré probado que estás excomulgado ${ }^{26}$. Más allá de la aparente puerilidad del ejemplo, la lógica que lo gobierna es la de la fragmentación del enunciado en proposiciones sucesivas no complejas. Pero existen otros elementos que pueden pertenecer a la experiencia de un hecho que la teoría de las positiones aconseja excluir. Hay en efecto positiones que no son pertinentes, otras que son superfluas, otras contradictorias. Por ejemplo, « pongamos que eres descomulgado porque feriste a clerigo, e despues faz otra posiçion e diz : "pongo que lo feriste en la cabeça" » tal posición se considera superflua dado que «quanto a la excomunion tanto es de ferirlo en la cabeça como en la mano o en cualquier otra parte ${ }^{27}$.

12 Las distinciones que pautan y reformulan los relatos son fundamentales. Es en ellas que reside una de las formas radicales de alteridad de los textos alfonsinos frente a los fueros. Lo que el Espéculo y las Partidas ofrecen es un marco de pensamiento, un método que debe organizar el conocimiento independientemente del objeto del proceso, como el resultado de una serie de distinciones sucesivas de orden lógico. Los fueros, por su parte, proporcionan correspondencias entre acciones y decisión judicial a partir de casos concretos, sin enunciar muchas prescripciones de método. En los fueros, el vínculo no se establece entre objeto de la prueba y medio de prueba, sino entre objeto del proceso y decisión judicial. Los fueros no construyen un marco metodológico, establecen más bien una taxonomía de causas en la que a cada una de ellas, definida de antemano, corresponde una pena estimada previamente.

Los textos alfonsinos postulan una suerte de focalización, de fragmentación de la complejidad de lo real de manera de construir, discursivamente, objetos " puros ». Al contrario, los fueros parecen mirar de lejos y reencontrar en un escenario complejo, pero indivisible, un suceso que la prueba debe manifestar, íntegramente. Los medios de prueba son, sin embargo, esencialmente los mismos en los dos corpora. No es allí, como hemos visto, donde reside la diferencia. Pero si el escenario se fragmenta, con él se fragmenta la prueba misma, que progresa hacia una lógica matemática que termina de afirmarse a fines del siglo XIII. Las Partidas y, más aún, en cierto modo, el Espéculo, proponen evaluar los hechos según un modo de filtrado de lo real en función de marcos dialécticos. Las distinciones que los jueces deben poner en ejecución de modo de circunscribir la demanda, y que están ausentes en los fueros, siguen a la litis contestatio, y definen la causa según una lógica compleja que no es la de la transparencia a lo real.

14 En los fueros, la prueba es sólo un medio para establecer una relación entre un hecho y una respuesta, que puede tomar la forma de un castigo, pero en las Siete Partidas y el Espéculo la prueba parece tomar gusto por multiplicar sus formas y sus incesantes fragmentaciones y recomposiciones. Si el lenguaje de los fueros es «realista» no es 
porque se busque un "efecto de realidad", sino porque se trata de textos que, brevemente y con ayuda de algunas fórmulas que designan la práctica, delimitan posibles situaciones de experiencia. En este gesto de codificación, instituyen un corpus de faltas ya identificadas, que las pruebas masivamente manifiestan o anulan. En el Espéculo y las Siete Partidas las distinciones de lenguaje, en la posición de la causa y en la recepción de las pruebas, alejan por el contrario el proceso del escenario de su «realidad» primera y rompen con el recorte preestablecido de la experiencia en formas fijas, para proponer otro que se establece en función de una serie de criterios lógicos sucesivos. En las Siete Partidas, la prueba ya no está solamente para ser útil, sino que se transforma en estrategia cognitiva compleja que impone a su vez una lectura de la realidad que depende de la precisión y la complejidad de la operación judicial.

Formas de creer

Las Siete Partidas instituyen la idea, propia del procedimiento romano-canónico, de que el «hecho" no puede ser conocido en su totalidad, que es irreductible a su reconstrucción. La verdad alcanzada en el marco del proceso no se fundamenta en una correspondencia absoluta entre la descripción judicial y las cosas tal y como sucedieron en el mundo real. Esta verdad es construida como una ficción, en la medida que -los juristas siguen en esto a Aristóteles- no existe certeza demostrativa acerca de lo contingente. Incluso el conocimiento directo de los hechos no exime de las operaciones necesarias para establecerlos. Es sólo al cabo de un proceso pautado en diferentes " tiempos " que se efectúa la verdad judicial ${ }^{28}$ : sólo la sentencia del juez produce la verdad de los hechos. La noción misma de prueba depende a la vez de la voluntad de restituir la presencia de los hechos ausentes con el propósito de volverlos perceptibles y de la conciencia de su imposible repetición. Esta conciencia de la separación entre la reconstrucción judicial y el hecho mismo, esta falta de lo real - como dice Michel de Certeau acerca de la escritura de la historia ${ }^{29}$-, esta distancia nunca borrada son los fundamentos de la verdad específica que nace del proceso. La idea de « cosa dudosa » (probatio est rei dubiae...) está inscrita en el centro de la prueba como el signo de esta separación, como la marca constitutiva de la verdad que funda.

Duda y argumenta

16 Entre los siglos XII y XIV dos sistemas se oponen en la definición de prueba. La primera distinción concierne el sentido que se otorga a la palabra argumenta. Esta puede ser entendida como definición genérica de toda prueba, o bien abandonada en favor de una enumeración : testigos, actas, indicios o presunciones. Para Gofredo de Trani (†1245), el uso de la palabra argumenta sitúa de antemano el proceso en el marco de la disputatio y está demasiado cerca del hallazgo agudo, ingenioso ${ }^{30}$. La prueba judicial debe hacer uso de testigos, documentos, indicios y presunciones. En este sentido, la palabra argumenta da demasiada importancia a lo que Aristóteles en su Retórica designa como las pruebas " artificiales ", provistas por el orador, independientemente de todas las que en efecto contienen rastros del hecho: testigos, contratos, declaraciones obtenidas bajo tortura, juramentos, que llama pruebas «no artificiales $»^{31}$. La segunda opción, elegida por las Partidas, separa prudentemente, como lo había hecho Gofredo de Trani, la noción de argumenta, criticada por los canonistas ${ }^{32}$. Los textos del rey Sabio no adoptan la noción de argumenta. Sin embargo, retoman las distinciones propias de una "verosimilitud» fundada en la lógica del relato.

Otra distinción fundamental lleva a pensar el concepto mismo de prueba en función de lo que es intrínseco o extrínseco al hecho. Si se define la prueba como lo que es 
extrínseco al hecho sólo hay prueba en la duda, es decir cuando el objeto del litigio no se muestra como ya resuelto, evidente. Es entonces que la prueba produce la luz de la verdad sobre un hecho que permanecía en la sombra. Si, por el contrario, se la define en función de lo que es intrínseco, la evidentia rei aparece como la prueba absoluta, resplandeciente y perfecta. En esta última opción, el notorium, o sea la ausencia de toda duda posible, es la mejor de las pruebas. Es así que en el siglo XIV Baldo (†1400) critica a Nicollaus de Matarellis ( $† 1310)$ que consideraba que la prueba es «extrinseca demonstratio rei dubiae per modos a iure statutos " porque, según él, la idea de una demostración extrínseca es fundamentalmente errónea. En tanto que Matarellis proponía una clasificación de medios de prueba donde la certeza absoluta del hecho que se constata está más allá de la prueba como su exceso - «Excedunt, complent, mediant, prosuntque probandi»-, Baldo pone en lugar de este exceso lo que considera como la mejor prueba, la "probatio per rei euidentiam est optima, et superlatiua probationum ${ }^{33}$. En las Partidas en cambio se encuentra la definición, que domina durante el siglo XIII, de la prueba como necesariamente ligada a la duda, a lo probable. Allí donde ninguna duda subsiste, incluso reducida al mínimo, no existe " prueba ». En su período clásico, la prueba preserva el rastro de esta falta que la constituye. Es en ella donde se enraíza la elaboración de una verdad cuya naturaleza no es ser evidente, sino resultar de una serie de operaciones que incluyen la acumulación de tipos de prueba disímiles que, aun sin poder alcanzar, como dirá Baldo, « una perfección unívoca » ${ }^{34}$, sirven, como sirve para el trabajo la mula, ese animal anómalo que no engendra. La prueba no es una manifestación, como la ordalía, ni la fijación de un hecho dotado de autonomía, como lo querrá la lógica de la prueba moderna ${ }^{35}$, sino una reconstrucción, consciente de dejar en el «hecho" reconstituido las huellas de la operación que lo establece.

Modalidades de la creencia

18 Desde la perspectiva en que la prueba sólo existe porque está ligada a la duda y supone la recolección de huellas de los hechos, las articulaciones de la creencia se tornan centrales en la gestión de justicia. Es necesario recordar que el ius commune afirma la necesidad de que el juez pronuncie la sentencia según las pruebas alegadas y no según su conciencia. Tomás de Piperata, en su tratado De fama, redactado entre 1265 y 1282, encarna la excepción proclamada a esta regla en tanto considera que el juez podía ejercer su arbitrium y fundar la sentencia en pruebas circunstanciales suficientes ${ }^{36}$. Los mecanismos de la creencia revelan en efecto la tensión que existe entre pruebas aportadas y discreción del juez. La construcción de la verdad se apoya en la acumulación de enunciados que no pueden escapar a su subjetividad inicial. Los textos jurídicos deben producir dispositivos destinados a contener los efectos de incertidumbre. La objetivación consiste pues en el hecho de dar a la mirada de un sujeto particular, el juez, el estatus de un « ojo teórico », pero no se debe olvidar que los glosadores retoman siempre el famoso rescripto de Adriano que recuerda que no existe regla certera que permita al juez evaluar los testimonios ${ }^{37}$. La construcción de un lugar objetivo pasa pues por la designación de una función pública. Ella borra el proceso subjetivo que lo condujo a la sentencia. En la Summa theologica, Tomás de Aquino limita el alcance de la conciencia del juez en la sentencia en función de un principio de exterioridad que realiza doblemente la verdad producida por el ordo: un principio común, que es el de las leyes contra las que ninguna prueba es aceptada, y un principio particular, que es el de los diferentes instrumentos de prueba en cada caso preciso. En 
tanto que persona, el hombre conoce según su conciencia, pero investido de una " publica potestas ", sólo puede conocer lo que se produce « in publico iudicio " ${ }^{38}$.

La confrontación de testimonios tanto como los criterios de admisibilidad de testigos permiten al juez equilibrar razonablemente lo que, en última instancia, esta obligado a hacer : creer en la palabra del prójimo. Esta forma de creencia, tan importante en san Agustín que afirma que no se debe rechazar lo que hemos sabido por el testimonio de otros, sino no se sabría que existe un océano, ni que existen las ciudades, ni dónde o de quién hemos nacido ${ }^{39}$, funda la verdad judicial. El marco del proceso excluye de antemano la creencia no fundada en las pruebas, como toda palabra que no emana de una boca digna. Pero si la creencia a priori y sin fisuras no tiene lugar en el proceso, es en torno de diferentes registros del creer que el conjunto del método se articula. Esto no carece de paradojas, no solamente porque la creencia puede llegar a significar incertidumbre, la expresión de un simple rumor, sino también porque creencia y saber son para el pensamiento medieval dos formas distintas de conocimiento, aunque ambas pueden producir verdades. Como en el derecho común, toda la lógica de prueba en los textos alfonsinos es la de la creencia y los criterios que permiten creer en los dichos de los testigos, determinar la credibilidad de las piezas escritas, fijar el grado de certeza que los testigos transmiten diciendo que saben o, por el contrario, que creen.

En un artículo publicado en 1999 he indicado tres modalidades de creencia que aparecen en los textos y que solo mencionaré brevemente ${ }^{40}$. La primera está determinada por el modo de percepción sensorial : se dice que se cree lo que se escuchó, en tanto que el saber reside en la visión. Esta primera modalidad de creencia concierne tanto al juez como a los testigos. La segunda modalidad de creencia remite a la adhesión del testigo - o sea de quien habla - a una posición. Pero en el interior de esta figura se pueden distinguir dos formas inicialmente distintas: la que privilegia la verdad interior, la fidelidad del individuo a su propia alma, y la que da consentimiento a lo que comúnmente se dice, a la fama hominis quoad alios. La tercera modalidad de creencia se sitúa del lado del juez, de quien escucha y se esfuerza por ver. Refiere al proceso que construye en él la adhesión fundada, la certeza probable de estar frente a la verdad. No me detendré en esto. Sólo quisiera recordar que las tensiones entre creencia y saber constituyen, a la vez, el eje de la construcción de la verdad en el espacio judicial y uno de los criterios de validación y verificación del testimonio, criterios a los cuales quisiera referirme para concluír.

Posibilidades del conocimiento y validación de los relatos

21 En el caso de los testigos, puede distinguirse en los textos castellanos del siglo XIII cinco criterios mayores de validación y de verificación en la construcción de la verdad. Es al interior de estos criterios que recorren el camino que va de las posibilidades del conocimiento a la validación de los relatos, que se inscriben las aceptaciones y las exclusiones del testimonio. Un primer criterio es el de la percepción sensorial y la preferencia por los testigos de visu. Pero, en segundo lugar, esta preferencia está fuertemente limitada por la asignación identitaria y la legitimidad de las declaraciones en función de la persona del declarante. En tercer lugar, la idea de conocimientos fundados en el modo de vida, o en los saberes prácticos, justifica que las mujeres, por ejemplo, cuyo testimonio es inválido en numerosos casos, puedan deponer en conflictos delimitados por espacios de sociabilidad, o en cuestiones de virginidad, embarazo y violación. Un cuarto criterio, que ya hemos expuesto, es el de las diferencias y similitudes entre saber y creencia. Y, finalmente, debe tenerse en cuenta la lógica 
interna de los discursos y la confrontación de los testimonios. De lo expuesto se desprende el recorrido que inscribe las particularidades propias del proceso judicial castellano del siglo XIII en un análisis de conceptos, de categorías y de representaciones que, en Occidente, fundan los criterios de la prueba y los modos de certificación.

Lo que ver quiere decir

El primer criterio concierne enteramente la posibilidad del conocimiento, las certezas y los errores de la visión. La preferencia de la Edad Media por la «voz viva» de los testigos como forma de prueba se apoya en la primacía dada por Aristóteles a los sentidos en los procesos cognitivos. Los grandes comentaristas del siglo XIV retoman la máxima escolástica - que da cuenta de los Segundos analíticos, I, 18.81 a 38 en los que Aristóteles establece la necesidad de la sensación para la inducción y la demostración : «Nihil est in intellectus quod non prius fuerit in sensu » ${ }^{41}$.

En el marco de esta primacía sensorial, la vista ocupa el primer lugar. La visión directa es considerada transmisora de una certeza cognitiva. Antes de los inicios del siglo XIV, no se encuentran desarrollos sobre la idea de que los sentidos pueden engañarnos. Pierre d'Auriol parece haber sido el primero en hacer de ello un tema de reflexión. Pero las equivocaciones del saber adquirido por los sentidos dependen, por una parte, de la complejidad del proceso " sensista ", que no acaba definitivamente sino en el intelecto, y por otra parte, de los problemas ligados a todo aquello que no depende de la visión directa : sólo ésta reúne en efecto las condiciones del conocimiento verdadero.

En el proceso sensible, con la sola percepción visual, nada se sabe todavía de lo que se ve fuera de los colores y las luces dispuestas según un determinado orden. La visión consiste en la recepción de una forma que se fija ante todo en la superficie externa del cristalino, luego es transmitida al cuerpo sutil, que se encuentra en la concavidad del nervio, para pasar enseguida a la parte común del nervio (el quiasma). Pero aun nada se sabe mientras que lo que se ve no ha sido aprehendido por otros sentidos. Sensibles comunes se agregan en efecto a lo que cada sentido proporciona en sí mismo, y su apreciación no puede operarse más que en el nivel de un «sentido común » que los compara, los selecciona y los juzga ${ }^{42}$.

La complejidad de la percepción y de la visión indirecta determinan formas inestables de la verdad transmitida por este sentido, incluso cuando se parte de la idea que está primero en el conocimiento. Tanto la complejidad de los procesos visuales comprendidos como procesos interpretativos, como las ilusiones producidas por todo lo que no coincide con la visión directa, cuando nada se interpone entre el objeto y la mirada, pueden hacer vacilar la certeza, o la identidad perfecta de dos percepciones. Haber visto no es jamás a fin de cuenta una prueba ex se. Solo la identidad autoriza las palabras de quienes tienen una boca digna de ser creída. Para los otros está la tortura, que arranca y construye los relatos de la "percepción sensorial» según formulas elaboradas por los jueces. El conocimiento se encuentra así doblemente determinado, por la complejidad de los procesos cognitivos, que conciernen a todos los seres humanos, por el lugar social de quienes testimonian.

Identidades

Para reconstruir un hecho, para validar las narraciones de lo que los testigos vieron, el juez debe fijar las identidades que determinarán la «fuerza » - el término es empleado por el Fuero Real a propósito de la estimación del testimonio de mujeres -, el índice de verdad que puede atribuir a cada enunciado. No solamente los fueros y el derecho alfonsino, sino también el derecho común en general y la tradición bíblica rechazan 
testimonios en función de la identidad de los testigos. El criterio de los fueros es en general el de la vecindad, que implica la residencia en una villa durante un período de al menos un año y un día teniendo "casa poblada ", un patrimonio suficiente y la exención de algunas « pechas ».

En las Partidas, la lista de excluidos del testimonio es imponente ${ }^{43}$. Algunas categorías resultan de lazos de afecto, de odio o de subordinación y, sobre todo, de acciones de los hombres que los textos alfonsinos reagrupan en torno de las ideas de mala fama, de error y de pecado. El juez debe así, en primer lugar, saber quién habla, pero sin que su evaluación sea verdaderamente subjetiva. Los textos ofrecen los diferentes criterios que le permitirán pesar los testimonios como si no decidiera -en el sentido pleno de la decisión. Exactamente lo mismo que para la verdad del hecho ausente, ellos transmiten la conciencia de la imposibilidad de aprehender, sin fallas, la complejidad de lo real. Los criterios de evaluación de los hombres jamás poseen las condiciones de una objetividad plena ${ }^{44}$, aunque la idea de una permanencia de la conducta permite decidir el futuro en función de lo que se sabe del pasado porque " consuetudo quasi altera quaedam natura ${ }^{45}$.

La fijación de identidad que filtra el relato asignándole grados variables de creencia en función del locutor depende, evidentemente, de una coherencia jerárquica. Pero este gesto no se reduce a una pura construcción ideológica de la verdad. Un hecho puede ser percibido por los sentidos de cualquier individuo - esto está fuertemente probado por la aceptación de todo testimonio cuando se trata de salvar al rey. Pero el conocimiento es diferente según la condición : ningún cuerpo puede sustraerse a la identidad social que piensa poseer ni a la que se le asigna. Estas determinaciones, que el derecho intenta fijar como globalmente estables, son un filtro cognitivo y narrativo en la reconstrucción de la percepción sensorial, no porque siempre arrojen sombra, sino porque iluminan diversamente la percepción del hecho pasado.

Modos de vida y saberes específicos

Algunos fueros, el Fuero Real y las Leyes de Estilo coinciden en el hecho de que no se pueden aceptar los testimonios femeninos sino en los litigios de mujeres o en los que una mujer al menos este directamente implicada. Esta decisión toma en cuenta el contexto del hecho a probar. Cuando uno de los manuscritos del Fuero Real resume en el acápite el contenido de la ley donde figura la cuestión de la aceptación de los testimonios de mujeres, menciona los « lugares » que le otorgan pertinencia - «En que lugares pueden testiguar las mugeres ${ }^{46}$. Hace referencia a lugares fijos, como el horno o el lavadero, pero también a situaciones de sociabilidad estrictamente femeninas, o bien a aquellas en las que las mujeres tienen un rol necesario y preponderante. Dentro de estos limites, pueden hacer una deposición cuya pertinencia revela la intuición del sentido, o su saber sobre prácticas e intercambios sociales específicos, lo que califica el relato de manera positiva en el proceso de reconstrucción del hecho. Aún cuando su percepción es a menudo descalificada como difusa, lo que limita -salvo en las Partidasla posible verosimilitud de lo que digan, se puede fiar de ellas cuando hablan de situaciones que la sociedad considera como experiencias típicamente femeninas, y sus saberes del cuerpo, tan importantes en la comunidad, se encuentran legitimados en el espacio judicial.

Es en particular el caso de las parteras, cuyo testimonio, en el derecho común, no está precedido por un juramento de veritate, sino de credulitate, es decir, el que privilegia la identidad entre lo que se dice y lo que se cree, mientras que los testigos ordinarios deben decir sólo lo que vieron y oyeron sin interpretación subjetiva del enunciado. Hay 
pues testigos que vieron y que dicen lo que saben, y otros que dicen lo que creen y que en algunos casos como el de las parteras, deponen sobre aquello que aun respondiendo a la percepción sensorial, depende efectivamente de un saber práctico que es la condición de la percepción. Aceptar la existencia de saberes específicos, fragmentarios y no sistemáticos, implica la aceptación de la relatividad, tanto de la noción de " hecho ", como de los fundamentos inestables de una verdad necesariamente plural. Si el saber está ligado a la visión, la creencia se sitúa del lado de los conocimientos conjeturales. Pero tanto uno como otro son relaciones posibles entre el hecho y la evidencia, y el primero no produce más transparencia a lo real que la segunda.

Creer y saber

31 Hemos visto ya que la noción de creencia está en el centro de la construcción judicial. Los textos vuelven constantemente sobre las maneras de fijar quién debe ser más creído, o menos creído que otros. El Espéculo IV, 7, 30 tiene por título: «A quales testigos deue el juez mas creer quando acaesciere dessacuerdo en ssus dichos ", y el texto de la ley acumula una serie de criterios de creencia que se apoyan ante todo en la calidad y la cantidad de testigos, luego en la verosimilitud del relato, que permiten construir la verdad como el resultado de la acumulación de fragmentos, de huellas de la realidad.

Pero esta creencia que construye por acumulación la verdad es múltiple, tanto en los fundamentos como en los grados de convicción que transmite. Todos los textos del rey Sabio prohíben la práctica de cojuradores, corriente como prueba en los fueros - sobre todo para el demandado cuya culpabilidad no haya sido probada por testigos visuales. Los que juraban así con el acusado no hacían sino atestiguar su valor moral, la imposibilidad abstracta de que hubiera cometido el hecho del que se lo acusaba. Los cojuradores se situaban así de entrada en una creencia enteramente independiente del hecho. El Espéculo y las Partidas exigen, al contrario, una creencia que debe siempre estar en relación con la memoria, el conocimiento del hecho - o al menos de un hecho que pueda servir como indicio inferencial. La primera condición puesta por el Espéculo IV , 11, 29 para evitar el perjurio es que « ssepa bien ciertamjente, o cree, que assi es lo que jura o non es assy. Et este ssaber dezimos que deua sseer, veyendo lo o acertando sse en ello. Et el creer otrossy deue sseer, aujendo ende tales ssenales o sseyendo la cosa que oyere tan con rrazon por que aya de creer, maguer non la vea. "

Las Siete Partidas 3.16.29, al final de la enumeración de casos donde el testimonio de auditu es aceptado, concluyen diciendo que la deposición de aquel «que non diere razon de cómo sabe lo que atestigua, si non que dize que lo cree, que non deue valer aquello que atestiguare ». Según la glosa de López la creencia no prueba, sino que ratifica. Pero si creencia y saber aparecen en el jurista imperial como dos formas que no participan de una misma esencia, en el siglo XIII, es el proceso de acumulación de creencia que conduce a la verdad judicial. A fines del siglo XV, la fides o credulitas que da acceso a las verdades probables, las únicas que la prueba clásica cree poder establecer, parece insuficiente para la construcción lógica y ritual de la verdad judicial. Es necesario reconocer en el mundo de los « hechos » una autonomía objetiva que borra la « falta » con relación a lo real de la cual la prueba clásica era consciente y que la remitía a las verdades probables y al assensum ${ }^{47}$. La noción misma de fides había comenzado entre los canonistas del siglo XIII, un proceso de depreciación inaugurado por Henry de Suse ( $† 1271)$ en su Summa a las Decretales de Gregorio IX, que la transformaba en un « nomen non finitae qualitatis » según el esquema doctrinal del «nomen infinitum ». 
Relegada en la accidentalidad y la falsedad cognitiva, la fides justificaba la intervención del magisterio de la Iglesia para organizar el conocimiento del hombre, perdido en los mil predicados posibles de una fe sujeta a todos los accidentes ${ }^{48}$. Pero en el siglo XIII la creencia es todavía una noción rica en modulaciones, en potencialidades cognitivas diversas que fundan, según una escala variable, el relato de los que hablan y la adhesión de los que escuchan.

Relato, presunción y lugares comunes

El último criterio de validación es el de la relación que cada relato mantiene con la verdad desde el punto de vista de la narración, la manera en la que es reconstruida una realidad borrada pero cuyos contornos pueden ser restituidos a través de la voz de los testigos, del contenido de los documentos, de los indicios y de las presunciones.

La definición de la prueba como « rei dubiae per argumenta facta demonstratio » domina el siglo XIII. Ella contiene como hemos visto dos elementos esenciales. Por una parte, el que instala la duda en el corazón de la prueba, por otra, el sentido que se debe dar a la palabra argumenta. El segundo elemento, el uso del término argumenta es, como hemos dicho, problemático. Los textos alfonsinos siguen una definición próxima a la que ofrece Gofredo de Trani, que elimina prudentemente toda referencia a los argumenta en favor de una enumeración que incluye testigos, escritos, indicios y presunciones. Pero más allá de los términos elegidos, el Espéculo y las Siete Partidas elaboran una verosimilitud que es en gran medida dependiente de la lógica del relato y que descansa sobre tres fundamentos: la pertinencia de las pruebas para un "factum probandum " con contornos precisos y limitados, la confrontación de testimonios, y el sentido de la praesumptio.

Ante todo, en lo que concierne a la pertinencia de las pruebas en relación con el hecho a probar, puede señalarse que en el Espéculo IV, 7, 30, cuando las dos partes presentan, al mismo tiempo, testigos que se contradicen, el juez debe seguir a aquellos que son más honorables, o de "mejor vida o costumbres ", o "quales dellos sse acuestan mas ssus dichos a la verdat diziendo rrazon que tanga mas ssenaladanjente al ffecho; et ssegunt aquello deue judgar ». Pero cómo poner como una condición misma del procedimiento la existencia de lo que se trata justamente de establecer, a saber, la verdad del hecho? Hay en realidad dos maneras de circunscribir la pertinencia de las pruebas aportadas. Por una parte, es en la narratio y la responsio que se encuentran diseminados los rastros de la verdad que el juez debe identificar. Por otra parte, la definición de un status - el hecho a probar - como "centro de argumentación » y criterio de pertinencia de las pruebas hace que sea posible situar la verdad que se trata de construir, no dentro de la idea de un hecho real y puramente objetivo que sería posible restituir, sino en la relación entre el hecho que debe ser probado, construido como una quaestio, y las pruebas que verifican la validez de su institución discursiva ${ }^{49}$. El hecho que debe ser probado es, a la vez, el producto de una selección de los rastros de lo real y una serie de distinciones discursivas.

El segundo criterio de verosimilitud del relato es la confrontación de testimonios. Se organiza en el Espéculo IV, 7, 19 según cinco « cosas » que deben coincidir. La primera es la « cosa » y el «ffecho » : el ejemplo dado es el de los objetos en juego, ya sea el objeto del litigio - se reclama una casa o una viña ? - o un objeto que entre de manera esencial en el asunto - se ha sido golpeado con una piedra o un bastón? La segunda, son las " perssonas de los omnes ». La tercera concierne al grado de parentesco cuando es pertinente. La cuarta es el lugar. Y la quinta es el tiempo, donde es necesario al menos 
distinguir entre la cosa que no puede suceder sino una vez - la muerte, la violación de una virgen o la pérdida de un miembro -, para la cual no puede haber desacuerdo, y la que admite la repetición y para la cual la no coincidencia de testimonios no conduce a su invalidación - el adulterio, la fornicación, la herida, el robo, etc.

Las Siete Partidas 3, 16, 28 no hacen una enumeración sistemática. Hablan del tiempo y del lugar, de la presencia de otros testigos del hecho, y sugieren que si el testigo es un hombre honorable, el juez no debe ir más lejos. Es solamente si está frente a un hombre «vil » que debe formular preguntas acerca del tiempo que hacía, el tipo de vestimenta que la gente llevaba, y otras todavía que permitan descubrir la posible mentira. A propósito de esta ley, la glosa de López aboga, con ayuda de santo Tomás, por una coincidencia de sentido que ignora los detalles del relato. La verdad no debe ser buscada en una coherencia obsesiva en la que la exactitud de detalles menores conduzcan a la credibilidad. El olvido de algunas cosas es natural a los hombres, que no intentan retenerlo todo en su memoria ${ }^{50}$.

El tercer criterio de evaluación de los relatos de las partes y de los testigos es el de la praesumptio, que al igual que los indicia, de los que no siempre se distingue claramente, mantiene una relación estrecha con la tradición clásica del ars dispuntandi ${ }^{51}$. La praesumptio implica una idea de lo que es normal, probable, que se funda en una probabilidad moralmente orientada. Es la opinión común la que determina la probabilidad, y no la frecuencia de un hecho. La praesumptio descontextualiza y fija la complejidad de conductas produciendo, a la vez, una serie de enunciados convencionales que determinan la verosimilitud de los relatos - la edad no cambia la naturaleza de una persona, los hombres poderosos no pueden tener miedo de los débiles, un padre no querría desheredar a un hijo para darle todo a otro, etc. -, y utilizando modos de argumentación ${ }^{52}$ que responde a la tradición de los Tópicos aristotélicos ${ }^{53}$. Cuando la aceptación de un argumento implica el reconocimiento de una cosa que parece poco probable o totalmente absurda de acuerdo con una normalidad ética, entonces el juez debe dudar de que ella conserve una relación con la verdad, a menos que sea capaz de probarla, y es aquí donde la praesumptio puede indicar sobre quien recae el peso de la prueba.

En un número reciente de los Annales. Histoire. Sciences Sociales consagrado a « Historia y Derecho ", Yan Thomas recuerda que las normas no se aplican directamente a los hechos mismos, "infinitamente diversos y polimorfos", como tampoco interpela, inmediatamente, a «sujetos irreductiblemente singulares». Las normas proyectan sobre los hechos sus propios contornos, designan sujetos « tan abstractos en relación a los individuos mismos como el sujeto gramatical en relación con la singularidad de cada uno de ellos ${ }^{54}$. En los trabajos resumidos aquí he intentado trazar un recorrido, discontinuo sin duda, pero tendiente a marcar formas especificas de los artificios jurídicos en un momento crucial de la historia de la normatividad occidental en el que aún producen una cierta extrañeza.

41 La contraposición entre la obra legislativa del rey Alfonso X y una coherencia previa, encarnada por los textos forales de la segunda mitad del siglo XII no implica, sin embargo, una contraposición entre una "costumbre " que emanaría de las cosas mismas y un derecho consciente de las ficciones que lo ordenan. Esto equivaldría a imaginar un orden jurídico que emana de la vida misma, "voz intacta de lo real», "dimensión que está en el interior de las cosas » ${ }^{55}$; momento anterior al advenimiento del artificio que sería no obstante, ya, un orden institucional. Los cambios que se 
imponen no constituyen uno de los tantos momentos de muerte anunciada de las identidades colectivas. No se trata de eso, sino de distinguir formas concurrentes de aprehensión y enunciación de la experiencia. Los ordines romano-canónicos obligan a prácticas de definición y restitución de los hechos que modifican clasificaciones anteriores que ya son jurídicas. Pero la tecnicidad y la complejidad crecientes crean un sector de especialistas cuya formación dependerá de nuevas instituciones. Los nobles « sabidores » del derecho serán remplazados por los técnicos del ius commune.

En el espacio judicial, los efectos de la recepción conducen a una verdad, que lejos de la inmanencia, resulta de una serie de procedimientos. Primero de calificación, de definición de "hechos" tratables. Luego de enunciación específica, es decir de establecimiento de los contornos de la causa en la litis contestatio y en las positiones. Finalmente, de aplicación de criterios de admisibilidad de las pruebas aportadas que movilizan identidades sociales, jerarquías cognitivas, modos de enunciación que el juez debe evaluar, preservando no obstante la regla que niega la afirmación de la conciencia individual. El proceso de designación y modelización de la experiencia conduce también a la redefinición de prácticas que son anteriores a su advenimiento, con el fin de asimilarlas a las instancias múltiples pero limitadas que el ordo impone. Reconocer las operaciones y categorías sucesivas que organizan la verdad no conduce a negar los hechos como fenómeno previo a la calificación, son ellos y no la verdad que pertenecen a la «exterioridad salvaje » ${ }^{56}$; Pero las operaciones que los formulan en el espacio judicial les han quitado inmediatez.

\section{NOTAS}

1. Este texto constituye una presentación resumida del libro Las verdades de los hechos. Entre fueros y obra jurídica alfonsina en la Castilla del siglo XIII, Ediciones Universidad Salamanca, 2004.

2. Cabe mencionar algunas de las empresas colectivas más significativas : J. CHANDLER, A. I. DAVIDSON y H. HAROOTUNIAN, Questions of evidence. Proof, practice, and persuasion across the disciplines, Chicago/Londres, 1994, que recoge una serie de artículos publicados por la revista Critical Inquiry entre 1991 y 1993 ; Annals of scholarship. An International quarterly in the humanities and social sciences, 8 (1991), "Rethinking objetivity », ed. A. MEGILl ; S. MARCHAND y E. LUNBECK, Proof and Persuasion. Essays on Authority, Objectivity, and Evidence, Bélgica, 1996.

3. C. GINZBURG, Rapports de force. Histoire, rhétorique, preuve, Paris, 2003 ; F. GOYET, Le sublime du « lieu commun ». L'invention rhétorique dans l'Antiquité et à la Renaissance, Paris, 1996 ; C. LÉVY y L. PERNOT, Dire l'évidence. Philosophie et rhétorique antiques, Paris, 1997 (Cahiers de philosophie de l'université de Paris-XII, 2).

4. S. SHAPIN y S. SCHAEFFER, Leviathan et la pompe à air. Hobbes et Boyle entre science et politique, Paris, 1993 ; S. SHAPIN, A Social History of Truth : Civility an Science in SeventeenthCentury England, Chicago, 1994 ; C. LICOPPE, La formation de la pratique scientifique. Le 
discours de l'expérience en France et en Angleterre (1630-1820), Paris, 1996 ; A. BLAIR, The Theater of Nature. Jean Bodin and Renaissance Science, Princeton, 1997.

5. El debate sobre las relaciones entre historia y ficción ha ocupado un lugar fundamental en la epistemología de la disciplina durante los últimos treinta años y da ya pruebas de un cierto agotamiento. Sería inútil y difícil resumir aquí la bibliografía sobre el tema, por otra parte ya bien conocida.

6. Sobre el proceso en Castilla y León entre los siglos XII y XIII, cabe destacar los trabajos de M. PAZ ALONSO ROMERO, El proceso penal en Castilla. Siglo XIII-XVIII, Salamanca, 1982 ; J. VALLEJO, « La regulación del proceso en el Fuero Real », Anuario de Historia del Derecho Español, 55 (1985), p. 495-703 ; A. GARCíA Y GARCíA, « El proceso canónico en la documentación medieval leonesa ", in El Reino de León en la Alta Edad Media. II. Ordenamiento jurídico del reino, León, 1992, p. 567-658 ; A. PRIETO MORERA, « El proceso en el reino de León a la luz de los diplomas », in El Reino de León..., ibid, p. 381-518. Con errores, pero de utilidad, J. CERDÁ RUIZ-FUENTES, « En torno a la pesquisa y procedimiento inquisitivo en el derecho castellano-leonés de la Edad Media », Anuario de Historia del derecho español, 32 (1962), p. 483-517.

7. Sobre las primeras fases de renacimiento jurídico, ver E. CORTESE, Il rinascimento giuridico medievale, Roma, 1992, como introducción general, del mismo autor, Il diritto nella storia medievale, 2 vol., Roma, 1995-1996.

8. En cuanto a la recepción en España, en Cataluña en primer lugar durante el primer tercio del siglo XII, ver A. GOURON, « Aux origines de l'influence des glossateurs en Espagne ", Historia, Instituciones y Documentos, 10 (1983), p. 325-346, reeditado en Études sur la diffusion des doctrines juridiques médiévales, Londres, 1987. A partir de los años 60 del siglo XII la influencia boloñesa se ejerce directamente, pero existen huellas de la influencia de las escuelas provenzales ya en el primer tercio del siglo XII, la primera sería de 1128, ver A. IGLESIA FERREIRós, « El primer testimonio de la recepción del Derecho romano en Cataluña? ", Revista jurídica de Cataluña 1978, p. 277 sq.

9. En los últimos años, la investigación sobre el derecho castellano se ha orientado hacia sus relaciones con el derecho común, siguiendo en esto un movimiento general de la historia del derecho en el ámbito europeo. Dos autores fundamentales que trabajan en esta perspectiva son Antonio García y García - ver en particular su Derecho común en España. Los juristas y sus obras, Murcia, 1991 -, y Antonio Pérez Martín - de su extensa obra de edición e investigación cabe señalar un texto central para nuestra problemática, El derecho procesal del « ius commune » en España, Murcia, 1999. No he podido desgraciadamente consultar el trabajo de E. MONTANOS FERRíN, España en la configuración histórico-jurídica de Europa, t. 1 (Entre el mundo antiguo y la primera edad medieval), Roma, 1997 y t. 2 (El Derecho Común y los Derechos Propios), Roma, 1999. 10. Son de particular interés los textos de R. M. FRAHER, «IV Laterans's Revolution in Criminal Procedure : The Birth of Inquisitio, the End of Ordeals, and Innocent III's Vision of Ecclesiastical Politics ", in R. J. CASTILLO LARA, Sudia in honorem eminentissimi cardinalis Alphonsi M. Stickler, Roma, 1992, p. 97-111 y J. THÉRY, « Fama : l'opinion publique comme preuve judiciaire. Aperçu sur la révolution médiévale de l'inquisitoir (XII ${ }^{\mathrm{e}} \mathrm{XIV}^{\mathrm{e}}$ siècle) », artículo inédito que le agradezco haberme permitido consultar. Sobre la complejidad de la práctica en ámbito comunal ver M. VALLERANI, Il sistema giudiziario del comune di Perugia : conflitti, reati e processi nella seconda metà del XIII secolo, Perugia, 1991, y « Il potere inquisitorio del podestà. Limiti e definizioni nella prassi bolognese di fine 
Duecento ", G. BARONE, L. CAPO y S. GASPARRI, Studi sul Medioevo per Girolamo Arnaldi, Roma, 2001, p. 379-417.

11. La relación entre imposición de una justicia pública y construcción del Estado es un lugar común, sin darle a este sintagma la menor tonalidad despectiva. Pero la relación fuerte, casi brutal, que une procedimiento inquisitorio y poder del Estado, ha sido postulada par Jacques Chiffoleau en una serie de artículos importantes : « Sur la pratique et la conjoncture de l'aveu judiciaire en France du XIII ${ }^{\mathrm{e}}$ au XIV ${ }^{\mathrm{e}}$ siècle », in L'aveu. Antiquité et Moyen Âge, Roma, 1986, p. 341-380 ; " Dire l'indicible : remarques sur la catégorie de nefandum du XII ${ }^{\mathrm{e}}$ au XV ${ }^{\mathrm{e}}$ siècle ", Annales ESC, 1990, p. 289-324 y "Sur le crime de majesté médiéval », in Genèse de l'État moderne en Méditerranée, Roma, 1993, p. 183-213.

12. M. T. CLANCHY, From memory to written record. England, 1066-1307, Cambridge Mass., 1979.

13. En toda Europa se produce, en torno al siglo XIII, lo que Mario Sbriccoli denominó para las ciudades italianas - como « la emergencia de un orden penal público », "Vidi communiter observari : l'emersione di un ordine penal pubblico nelle città italiane del secolo XIII », Quaderni fiorentini per la storia del pensiero giuridico moderno, 27 (1998), p. 231-268, afirmación esencial del poder soberano. La visibilidad del castigo juega por otra parte explicitamente un rol de prevención, ver R. M. FRAHER, « Preventing crime in the High Middle Ages : the medieval lawyers'search for deterrence », in J. ROSS SWEENEY y S. CHODOROW, prólogo de S. KUTTNER, Popes, teachers, and canon law in the Middle Ages, Ithaca/Londres, 1989, p. 212-233.

14. M. BAXANDALL, Les humanistes à la découverte de la composition en peinture 1340-1450, Paris, 1989, p. 68.

15. M. BAXANDALL, Les humanistes..., ibid, p. 64.

16. Para una crítica a este tipo de enfoque, ver A. BOUREAU, La loi du royaume. Les moines, le droit et la construction de la nation anglaise (XI ${ }^{e}-\mathrm{XIII}^{e}$ siècle), Paris, 2001.

17. J. W. SCOTT, « The evidence of experience », in J. CHANDLER, A. I. DAVIDSON y

H. HAROOTUNIAN, Questions of evidence..., op. cit., p. 387.

18. He trabajado con un conjunto de fueros largos seleccionando algunos textos mayores y procurando cubrir un espacio geográfico amplio. El fuero de Cuenca, cabeza de familia de un grupo importante de concesiones, el fuero de Madrid, el de Coria - que se inscribe en una familia foral de la región gallego-portuguesa -, el fuero castellano de Soria, el de Sepúlveda, y el fuero navarro de Estella. Las ediciones utilizadas son las siguientes : R. UREÑA Y SMENJAUD, Fuero de Cuenca, Madrid, 1935 ; G. SÁNCHEZ, A. MILLARES CARLO y R. LAPESA, El Fuero de Madrid, Madrid, 1932 ; E. SÁEZ y J. MALDONADO Y FERNÁNDEZ DEL TORCO, El Fuero de Coria, Coria, 1949 ; G. SÁNCHEZ, Fueros castellanos de Soria y Alcalá de Henares, Madrid, 1919 ; E. SÁEZ, R. GIBERT y M. ALVAR, Los Fueros de Sepúlveda, Segovia, 1953 ; G. HOLMÉR, El fuero de Estella, Karlshamn, 1963.

19. Edición de G. SÁNCHEZ, Libro de los Fueros de Castiella, Barcelona, 1981.

20. Hemos utilizado las siguientes ediciones de la obra alfonsina : G. MARTínEz DIEZ, Fuero Real, Ávila, 1988 ; R. A. MCDONALD, Espéculo, Madison, 1990 y Las Siete Partidas del sabio rey don Alfonso el nono, nuevamente glosadas por el licenciado Gregorio López, edición de Salamanca, 1555 por Andrea Portonaris (reproducción del Boletín Oficial del Estado, 1974).

21. A. GIULIANI, Il concetto di prova. Contributo alla logica giuridica, Milán, 1971. 
22. Se trata de la determinación de positiones, que las Partidas llaman " preguntas » (Siete Partidas 3.13.2 « Pregunta es cosa de que nace grand pro. Ca por ella puede el judgador saber mas en cierto la verdad de los pleytos, e de los fechos dubdosos, que vienen ante el. E puede la fazer el juez, fasta que de el juyzio : e aun la vna parte ala otra, ante el judgador. E deue ser de tal natura, que pertenezca al fecho, o a la cosa sobre que es la contienda. E ha se fazer en cierto, e por pocas palabras, non emboluiendo muchas razones en vno. De manera que el preguntado las pueda entender, e responder ciertamente a ellas. Ca si de otra guisa fuesse fecha, non deue ser cabida : ni aun la parte a quien la fiziessen, non seria tenudo de responder a ella ») y de la realización de la litis contestatio.

23. QUINTILIANO, Institutio oratoria, III, 6, 21, ed. y trad. de J. cousin, Paris, 1976.

24. Joaquín Cerdá editó el texto en «La "Margarita de los pleitos" de Fernando Martínez de Zamora », Anuario de historia del derecho español, 29 (1959), p. 634-738. Se atribuye la autoría a Fernando Martínez de Zamora, autor de la Summa aurea de ordine judiciario editada por Antonio Pérez Martín (El derecho procesal..., op. cit., p. 133-215). En opinión de Antonio Pérez Martín nada justifica esta atribución, ver p. 50 de obra citada. El texto carece de originalidad, el tipo de advertencias que ofrece es común a varios textos del siglo XIII, ver M. VALLERANI, « I fatti nella logica del processo medievale. Note introduttive », Quaderni Storici, XXXVI/ 108 (2001), p. 665-693.

25. Sobre las positiones, M. VALLERANI, « I fatti nella logica... », ibid., p. 673 sqq.

26. J. CERDÁ, « La “Margarita... », op. cit., p. 719-720.

27. J. CERDÁ, « La "Margarita... », ibid., p. 721.

28. Siete Partidas 3. 22. 5, « [...] mas si el demandador e el demando non fuessen emplazados maguer que el sepa toda la verdad del pleyto non deue entonces el juzgar [...] ». La misma exigencia aparece en las Siete Partidas 3. 22. 22.

29. M. DE CERTEAU, La escritura de la historia, México, 1993 ( $2^{\mathrm{e}}$ ed.), p. 13.

30. GOFREDO DE TRANI, Summa in titulos decretalium, Venecia, 1570, X 2. 19, rúbrica ${ }^{\circ} 1$ « (...) dicitur argumentum quasi argutè inuentum. probatio autem testibus et fide tabularum constat. Vnde etsi haec diffinitio locum habet in his quae probantur per instantiam disputandi. non tamen locum habet in his quae probantur per testes vel instrumenta, et prelunque per praesumptiones et indicia. »

31. La palabra argumentum es ambigua puesto que para algunos designa tanto pruebas « artificiales » como pruebas « no artificiales ». Así en la Summa codicis atribuida a Irnerius (ed. H. FITTING, «Summa Codicis » des Irnerius, Berlín, 1894) C 4. 19.3 « Argumenta quidem sunt quae causam indicent atque ostendunt, et ideo testes et instrumenta item inditia sub hac appellatione continentur. » La glosa ordinaria dice : C. 4. 19 De probationibus «[...] Et est probatio, rei dubiae per argumenta ostensio, et dicitur secundum $\mathrm{P}$ [lacentinum] a probe aduerbio. probe enim agit, qui probat hoc, quod intendet. " Esta definición retoma lo dicho por Azon en su Summa sobre C. 4. 19. Sobre la relación entre prueba y retórica y la compatibilidad de ambos conceptos en Aristóteles, compatibilidad que se traduce en el sistema probatorio medieval, ver C. GINZBURG, Rapports de force..., op. cit., cap. 1.

32. BALDO DE UBALDIS, In Quartum et Quintum Cod. Lib. Commentaria, Venecia, 1599, C. 4.19 rúbrica $n^{\circ} 3$ y 4 « Probatio, est rei dubiae per argumenta ostentio. hanc diffinitionem reprehendunt Canonistae, dicentes que argumentum propriè est Artistarum, et Legistarum, non testium, vel Tabellionum. "

33. BALDO DE UBALDIS, In Quartum..., ibid, n² 20. 
34. BALDO DE UBALDIS, Ad Tres priores libros Decretalium..., ibid., X. 2. 19, rúbrica $n^{\circ} 3$ « Porro tunc ex coniuctione duarum semiplenarum probationum resultat vna species perfectae probationis, quando istae duae semiplenae sunt eiusdem forme et substantiae. Sed si sunt diuersorum generum [...] tunc est ibi cumulus praesumptionum, tamen quia sunt diuersae rationis et non cumulantur ad vnum communem esse non possunt producere aliqualem formam vniuocae perfectionis [...] Est enim probatio similis rebus corporeis in quibus diuisio continui non inducit perfectionem, sed aegritudinem et morbum [...] Argumentum tamen facit praesumptio praesumtioni, et resultat interdum quaedam probatio anomala sicut ex equa et asino resultat quoddam animal anomalum, quod non generat. id est mulus, et tamen multo est efficax in operando, permiscentur ergo species probationis non ad vnitatem perfectionis, sed ad validitatem praesumptionis. "

35. Esta tipología de diferentes modalidades formales de prueba es la que propone Alessandro Giuliani. Sin embargo, no creemos que se organice siempre siguiendo rupturas cronológicas, sino que es susceptible de designar formas que pueden ser utilizadas conjuntamente. En el siglo XVI aún, en pleno apogeo de lo que Giuliani define como la " prueba moderna ", el recurso a las ordalías en la práctica judicial no ha desaparecido ; en efecto, el recurso a la manifestación divina completa con frecuencia las instancias de la « prueba clásica » - es decir, la que según Alessandro Giuliani rige entre el fin del siglo XII y XV. Los historiadores que han trabajado sobre la práctica judicial, han mostrado en general que el cruce de prácticas y criterios es una constante. Ver por ejemplo los textos arriba citados de Massimo Vallerani, y la gran tesis de Claude Gauvard.

36. R. M. FRAHER, « Conviction according to conscience : the medieval jurists'debate concerning judicial discretion and the law of proof ", Law and History Review, 7 (1989), p. 23-88.

37. Digesto 22. 5. 3. 2 "Quae argumenta ad quem modum probandae cuique rei sufficiant, nullo certo modo satis definiri potest [...] Hoc ergo solum tibi rescribere possum summatim, non vtique ad vnam probationis speciem cognitionem statim alligari debere: sed ex sententia animi tui te aestimare oportere quid aut credas, aut parum probatum opineris. "

38. Summa theologica, IIa, IIae, q. 67, art. 2.

39. SAN AGUSTín, De trinitate, XV, XII, 21, Euvres, t. 16, texto de la edición benedictina, Paris, 1991 (Études augustinnienes).

40. M. MADERO, « Façons de croire. Les témoins et le juge et les témoins dans l'œuvre juridique d'Alphonse X le Sage, roi de Castille », Annales HSS (1999), p. 197-218.

41. BARTOLO DA SASSOFERRATO, Tractatus testimoniorum, éd. S. LEPSIUS, Der Richter und die Zeugen. Eine Untersuchung anhand des Tractatus testimoniorum des Bartolus von Sassoferrato. Mit Edition, Frankfurt am Main, 2003, $\mathrm{n}^{\circ} 4$ « secundum naturam nil est in intellectu quod prius non fuerit in sensu ». Ver también, BALDO DE UBALDIS, In Quartum et Quintum Cod. Lib..., op. cit., C. 4. 19., $\mathrm{n}^{\circ} 1$ « probatio per rei evidentiam est optima et superlativa probationum : quia illi sunt verissimili sermones qui concordant rebus sensatis, testibus Aristoteles et Averroes ».

42. G. SIMON, « Derrière le miroir ", Le Temps de la réflexion, t. 2, 1981, p. 318-319.

43. Siete Partidas 3. 16. 8.

44. D. 22. 5. 3. 2.

45. CICERÓN, De finibus, V. 74. Acerca de la naturaleza como « institución » en el derecho romano clásico, ver el remarcable artículo de Y. THOMAS, « Imago naturae. Note sur l'institutionnalité de la nature à Rome ", Théologie et droit dans la science politique de l'État moderne, Actes de la table ronde organisée par l'École française de Rome et le CNRS, Rome, 12-14 
novembre de 1987, Roma, 1991, p. 201-227. Sobre el adagio « consuetudo quasi altera quaedam natura » en la tradición jurídica occidental, ver D. R. KELLEY, The human mesure : social thought in the western legal tradition, Cambridge, Mass., 1990.

46. Ms. $P$, de Perelada, Gerona, Biblioteca del Palacio, ms. 14 984, de la segunda mitad del siglo XV.

47. A. GIULIANI, Il concetto di prova..., op. cit., p. 208.

48. M. MONTORZI, « Fides in rem publicam. » Ambiguità e techniche del diritto comune, Nápoles, 1984, p. 29-33. Nomen infinitum, es el sustantivo sin referente ontológico, que designa realidades negativas : nihil, nox, peccatum, silentium, non homo. Es pues un sustantivo que es un puro predicado, ver nota 45, p. 32

49. La teoría del status, de Hermágoras de Temno (I. a. C.), es así definida por Quintiliano: «Hermagoras statum vocat, per quem subiecta res intelligatur et ad quem probationes etiam partium referantur ", Institutio oratoria III. 6. 21.

50. Se trata de la Summa theologica IIae, q. 70, art. 2.

51. Sobre este tema, ver I. ROSONI, Quae singula non prosunt collecta iuvant. La teoria della prova indiziaria nell'età medievale e moderna, Milan, 1995, en particular, el capítulo III. 52. Sobre los modi arguendi in iure, ver M. BELLOMO, I fatti e il diritto. Tra le certezze e i dubbi dei giuristi medievali (secoli XIII-XIV), Roma, 2000, capítulo XI « Il metodo : fra logica e libri legales ».

53. Sobre este tema ver N. J. GREEN PEDERSEN, The tradition of the topics in the Middle Ages: the commentaries on Aristotle's and Boethius' Topics, Múnich, 1984.

54. Y. THOMAS, " Présentation », « Histoire et droit », Annales HSS (2002), p. 1426.

55. P. GROSSI, El orden jurídico medieval, Madrid, 1996, p. 108-109. Ver la crítica a esta postura hecha por E. CONTE, « Un débat historiographique italien », Annales HSS (2002), p. 1593-1613.

56. M. FOUCAULT, L'ordre du discours, Paris, 1971, p. 37.

ÍNDICE

Índice geográfico: Espagne/Castille

Mots-clés: preuve, témoignage

\section{AUTOR}

MARTA MADERO

Universidad Nacional de General Sarmiento 